\title{
Identification of Multi-fractal Characteristics of Silicon Content in Blast Furnace Hot Metal
}

\author{
Shi-hua LUO, ${ }^{1,2)}$ Xiang-guan LIU ${ }^{21}$ and Jiu-sun ZENG ${ }^{21}$ \\ 1) School of Information Management, Jiangxi University of Finance and Economics, Nanchang, 330013, China. \\ E-mail: Iuoshihua@nbu.edu.cn $\quad$ 2) The Institute of System Optimum Technique, Zhejiang University, Hangzhou, 310027, \\ China. E-mail: superman100f@yahoo.com.cn, zjs1020@hotmail.com
}

(Received on February 14, 2007; accepted on May 28, 2007)

\begin{abstract}
An improved approach was proposed to identify the multi-fractal characteristics of silicon content in hot metal from No. 7 BF at Handan Steel, No. 1 BF at Laiwu Steel and No. 6 BF at Linfen Iron and Steel Group Co. Comprehensive and quantitative details of the partial fluctuant singularity and fluctuant singularity at different levels are displayed through the computation of generalized Hurst index, scaling function and multifractal spectrum. The results confirm the existence of multi-fractal characteristics in the investigated time series. To describe such fluctuation in a single Hurst index or box dimension is insufficient. It is concluded that the theory and methods based on normal distribution, sequence unrelated and single fractal needs addition and amendments.
\end{abstract}

KEY WORDS: blast furnace iron-making; silicon content; multi-fractal; generalized Hurst index; multi-fractal spectrum.

\section{Introduction}

Blast furnace (BF) iron-making is a complex nonlinear process under high temperature and high pressure. More than 100 chemical reactions happen in the blast furnace simultaneously. The dynamic reactions under industrial conditions cannot be simulated in the laboratory completely. ${ }^{1)}$ The complexity of iron-making process has prevented it from being controlled optimally. To better control the ironmaking process, lower coke rate and increase utility, attempts have been made to develop BF expert system. During the past decade, researchers in Japan, Finland and China etc. have developed expert systems with unique features. $^{2-4)}$ All the expert systems developed share a common and important module: prediction and control of silicon content which represents the thermal state in the BF. Recently, several algorithms based on random process ${ }^{5-13)}$ are proposed to predict silicon content. But the existing models cannot work well due to the high fluctuation of silicon content.

To solve the problem, we take in-depth exploration for the cause of fluctuation of silicon content. By computation of Hurst index, box dimension and power spectrum of silicon content series, the existence of fractal characteristics has been confirmed, ${ }^{14,15)}$ the series is not random. However, the previous work described the fractal characteristics of time series using a simple parameter, e.g. Hurst index or fractal dimension. Only the single-fractal structure of silicon content series was discussed. Single-fractal structure deals with long-term statistical behaviors, it gives an overall picture of fluctuation but not the delicate structure in detail. But it is always not the case, practical production changes constantly with time and other conditions. To better describe the fluctuation of silicon content, we introduce the multi-fractal structure.

In the early $1980 \mathrm{~s}$, Grassberger ${ }^{16,17)}$ introduced the multifractal theory systematically. Generalized Hurst index, generalized fractal dimension, multi-fractal spectrum and other index are used to describe the multi-fractal structure. Much research effort has been contributed to the development of numerical methods to compute these indicators. R. Benzi, J. W. Kantelhardt, Racry E., Schmitt et al. ${ }^{18-20)}$ have done excellent work.

In this paper, the sample space is time series of silicon content in hot metal from No. $7 \mathrm{BF}\left(2000 \mathrm{~m}^{3}\right)$ at Handan Steel, No. 1 BF $\left(750 \mathrm{~m}^{3}\right)$ at Laiwu Steel and No. 6 BF $\left(380 \mathrm{~m}^{3}\right)$ at Linfen Steel, with the size of 1000 respectively. Generalized Hurst index, multi-fractal spectrum and other time variant parameters are measured to describe the multifractal characteristics of the thermal state in $\mathrm{BF}$, e.g., the irregularity or singularity of the fluctuation of silicon content. Simulation results show the local fluctuation and singularity is not consistent, which confirmed the existence of multi-fractal characteristics. This is a worthwhile result either in the terms of theory or in the practical application.

\section{Multi-fractal Analysis}

For a process proved to be single fractal, the existence of multi-fractal characteristics is tested through calculation of some generalized fractal parameters that are used to describe the local fractal characteristic of the process. Existence of multi-fractal characteristics is confirmed when the calculated parameters are time variant or extent variant. 
Multi-fractal process shows more complex and irregular fluctuation than ideal single fractal process. Here are some important generalized fractal parameters ${ }^{21-23)}$.

\subsection{Scale Function}

Definition 1. A stochastic process $\{X(t), t \in T\}$ with stationary increment is called a multi-fractal process provided that:

$$
E\left(|X(t+\mathrm{V} t)-X(t)|^{q}\right)=c(q)(\mathrm{V} t)^{\tau(q)+1}, \quad \forall t \in T, q \in Q \ldots
$$

$\tau(q)$ is the scale function or quality index, it is an important index of fractal. For a multi-fractal process $\tau(q)$ is a nonlinear function and the process described in (1) retreats to a single fractal process when $\tau(q)$ is linear. It is shown in (1) that the scale power law changes for different time increment $\mathrm{V} t$, e.g., the basic feature of scaling-invariance for a fractal process is satisfied. Multi-fractal describes the local singularity of fractal process. At different time points, a power computing magnifies or reduces the level of fluctuation. As a result, a sharp fluctuation becomes sharper and a small fluctuation becomes smaller. We'll further discuss the physical meaning of a multi-fractal process considering the two special cases:

(1) If $q<0$ and $|q|>10$, a relatively sharp fluctuation $|X(t+\mathrm{V} t)-X(t)|$ converges to zero after a power computing of order $q$. Thus the sharp fluctuation almost has no influence to the process while the small fluctuation dominates the process. The $q$ th order distance describes the small fluctuation feature of the process.

(2) If $q>0$ and $|q|>10$, the relative small fluctuation $|X(t+\mathrm{V} t)-X(t)|$ converges to zero after a power computing of order $q$. Thus the sharp fluctuation dominates the process. The $q$ th order distance describes the sharp fluctuation feature of the process.

\subsection{Generalized Hurst Index}

Stimulated by Einstein's research on Brownian motion, e.g. the proportional relation between the traversed path and time span: $R=T^{0.5}$, Hurst [1965] developed the rescaled range analysis, a statistical method to analyze long records of natural phenomena. There are two factors used in this analysis: firstly the range $R$, this is the difference between the minimum and maximum 'accumulated' values or cumulative sum of $X(t+\mathrm{V} t)$ for a natural phenomenon at discrete integer-valued time $t$ over a time span $\mathrm{V} t$, and secondly the standard deviation $S$, estimated from the observed values. Hurst found that the ratio $R / S$ is very well described for a large number of natural phenomena by the following empirical relation:

$$
(R / S)_{N}=C N^{H}
$$

where $C$ is a constant, $N$ the sequence length and $H$ the Hurst exponent. Extend this kind of thinking to the multi-fractal process we define the generalized Hurst exponent $H(q)$ :

$$
\left\{E\left(|X(t+\mathrm{V} t)-X(t)|^{q}\right)\right\}^{1 / q}=c(q)(\mathrm{V} t)^{H(q)}
$$

Generalized Hurst exponent and scale function $\tau(q)$ satisfy the following equation:

$$
H(q)=[\tau(q)+1] / q
$$

If an appropriate method is found to simulate the scale function $\tau(q)$, the generalized Hurst exponent $H(q)$ can be easily got. On the other hand, if $H(q)$ is calculated, $\tau(q)$ can also be got easily.

\subsection{Multi-fractal Spectrum}

Definition 2. Given a function $X(t)$ defined in a certain neighborhood of $t$, it follows that:

$$
|X(t+\Delta t)-X(t)| \sim C_{t}(\Delta t)^{\alpha(t)}
$$

when $\mathrm{V} t \rightarrow 0$, divide the interval $[0, T]$ into $b^{k}$ small subintervals and repeat this division. Let $N_{k}(\alpha)$ be the number of subintervals that include $\alpha(t)$ for the $k$ th classification section. Define a new function $f(a)$ as:

$$
f(\alpha)=\lim _{k \rightarrow \infty}\left(\frac{\log N_{k}(\alpha)}{\log \left(b^{k}\right)}\right) \ldots
$$

$X(t)$ is a multi-fractal process if the domain of $f(a)$ is positive and not just one point, where $a(t)$ is the Holder index and $f(a)$ is the multi-fractal spectrum.

In the above definition, local Holder index $a(t)$ describes the smooth or irregular fluctuation of a process in some local section; while multi-fractal spectrum $f(a)$ describes the probability of a randomly taken time point with Holder index $a(t)$ in a process. The most profound nature of multifractal is the diversity of local scale features, e.g., Holder index has a diverse range if the process has the feature of multi-fractal.

\section{Identification Algorithm Design}

The most commonly used method to identify a multifractal structure is to estimate the scale function $\tau(q)$ using an appropriate algorithm. From Eq. (4) we can get the generalized Hurst index $H(q)$ and the multi-fractal spectrum $f(a)$ can be generated by equation $f(\alpha)=\inf _{q \in R}\{\alpha q-$ $\tau(q)\} .^{24,25)}$ Most simulation algorithms of $\tau(q)$ are based on ergodic theory and thermodynamics. These methods have strong preconditions of time series being normal and strictly stationary, for time series from a practical industrial process, simulation results are not reliable.

Kantelhardt et al. proposed a new method of multi-fractal analysis - multi-fractal detrended fluctuation analysis, abbreviated as MF-DFA method, ${ }^{19)}$ which is an extension of DFA method. For non-stationary time series, the robustness of DFA has been widely recognized, so an extension of DFA is natural. What's more, Kantelhardt proved that using MF-DFA, the tag index is the same as that of standard multi-fractal methods for normal and stationary time series. With MF-DFA, we can get a new framework of multi-fractal identification. Here we give the new algorithms:

\subsection{Using MF-DFA to Estimate Generalized Hurst Index}

For a given time series of silicon content ([Si $]_{n}, n=$ $1,2, \ldots, N$ ) with $N$ samples, we'll take the following steps to estimate generalized Hurst index:

(1) Construct the accumulated deviation series $\{y(i)\}$ :

$$
y(i)=\sum_{l=1}^{i}\left([\mathrm{Si}]_{l}-\overline{[\mathrm{Si}]}\right) \quad i=1,2, \ldots, N
$$

where $\overline{[\mathrm{Si}]}=(1 / N) \sum_{i=1}^{N}[\mathrm{Si}]_{i}$; 
(2) Divide the series $\{y(i)\}$ into $m$ mutual exclusive sections, each containing $s$ data points. Problem arises that the tailed data may be emitted since it does not strictly follow that $m \times s=N$. To solve this problem we reverse the series according to the index and do the division again, so that for a given $s$ we can get $2 m$ small sections.

(3) For data points in each section $v(v=1,2, \ldots, 2 m)$, do $k$-order least square polynomial fitting and get the fitted functions:

$$
\overline{y_{v(i)}}=a_{0}+a_{1} i+a_{2} i^{2}+\cdots+a_{k} i^{k}
$$

(4) Compute the mean square error (MSE). $F^{2}(v, s)$. If $v=1,2, \ldots, m$, it follows:

$$
F^{2}(v, s)=\frac{1}{s} \sum_{i=1}^{s}\left\{y[(v-1) s+i]-\overline{y_{v(i)}}\right\}^{2}
$$

While for $v=m, m+2, \ldots, 2 m$, MSE is got through the following equation:

$$
F^{2}(v, s)=\frac{1}{s} \sum_{i=1}^{s}\left\{y[N-(v-m) s+i]-\overline{y_{v(i)}}\right\}^{2}
$$

(5) Introduce the $q$-order fluctuation function $F_{q}(s)$ to describe the mean of MSE in all sections:

$$
F_{q}(s)=\left\{\frac{1}{2 m} \sum_{v=1}^{2 m}\left[F^{2}(v, s)\right]^{q / 2}\right\}^{1 / q}
$$

The physical meaning of definition (11) is similar to the result of previous chapter. Order $q$ highlights the influence of different fluctuations to function $F_{q}(s)$. If $q<0$ and $|q|>10$, a relatively sharp error $F^{2}(v, s)$ converges to zero after a power computing of order $q$. The dominant influence is exerted by the small error and the influence of sharp error can be omitted and vice versa. The difference lies in the fact that during the process of generating $F^{2}(v, s)$, a polynomial fitting was used to smooth each section. Thus the influence of series being non-stationary is eliminated.

It should be noted when $q=0$, definition (11) doesn't hold. Since our attention is contributed to the correlation between $H(q)$ and $q$, the identification result won't change even if we neglect the values of $q=0$.

(6) If it holds that $\max (K+2,10) \leq s \leq N / 4$ and $F_{q}(s)$ is bounded by a power law growth of $s$ :

$$
F_{q}(s) \sim s^{H(q)}
$$

From (12) we can get $\lg F_{q}(s)=H(q) \lg s+C$, where $H(q)$ is the generalized Hurst index. $H(q)$ is estimated by fitting in the logarithm coordinate map. If the series is a single fractal process, it can be easily derived that $H(q)$ is a constant, and the existence of multi-fractal structure is proved if $H(q)$ is a nonlinear function of $q$.

\subsection{Estimate the Scale Function}

For a multi-fractal process given by definition (1), its generalized Hurst index $H(q)$ and scale function $\tau(q)$ follow the equation $H(q)=[\tau(q)+1] / q$. For the $H(q)$ estimated through MF-DFA (to avoid confusion we take it as $\overline{H(q)})$, it remains a problem whether the equation can be used to solve $\tau(q)$. We transform (12) to the following form:

$$
F_{q}(s)=\left\{\frac{1}{2 m} \sum_{v=1}^{2 m}\left[\sqrt{F^{2}(v, s)}\right]^{q}\right\}^{1 / q} \sim s^{\overline{H(q)}}
$$

And transform (3) into:

$$
\left\{E[|(X(t+\mathrm{V} t)-X(t))|]^{q}\right\}^{1 / q} \sim(\mathrm{V} t)^{H(q)} \ldots
$$

Noted that (12) and (13) have the same physical meaning, the difference lies in the numerical methods used to compute local fluctuation. Thus there exists a function $\varphi$ such that $\overline{H(q)}=\varphi(H(q))$. The linear or nonlinear relation of $H(q) \sim q$ remains the same under the function $\varphi$. So the definitions about generalized Hurst index $H(q)$ by (13) and (14) are the same. By formula (1), we can define the scale function $\overline{\tau(q)}$ afresh as:

$$
\left\{\frac{1}{2 m} \sum_{v=1}^{2 m}\left[\sqrt{F^{2}(v, s)}\right]^{q}\right\}^{1 / q} \sim s^{\overline{\tau(q)}+1}
$$

Contrasting (13) and (15), we can gain the equation:

$$
\overline{\tau(q)}=q \times \overline{H(q)}-1 \text {. }
$$

\subsection{Estimate the Multi-fractal Spectrum}

R. Benzi, G. Paladin, U. Frisch and other physicists suggested a new method to estimate the multi-fractal spectrum $f(a)$ of process $X(t)$. It is estimated via a Legendre transformation of scale function $\tau(q)$ :

$$
f(\alpha)=\inf _{q \in R}\{\alpha q-\tau(q)\}
$$

The transformation can be decomposed numerically, but a graphic description makes more sense. For each $q$ we can draw a straight line with slope $q$ and intercept $-\tau(q)$. With all the straight lines drawn in a graph, the bottom boundary gives the multi-fractal spectrum. If there is more than one point in the multi-spectrum we regard the process has multi-fractal characteristics.

\section{Simulation of Algorithm}

For the current study, data of silicon content in hot metal observed in Handan Steel, Laiwu Steel and Linfen Steel are used; the size of data is 1000 respectively, and the time of sampling interval is about $2 \mathrm{~h}$. All the work has been done on the software pack Matlab 6.5 (The Mathworks Inc., Natick, MA). The time series data as a function of Heat No. are presented in Fig. 1. For comparison, we generate a Gaussian random series with the same size using the randn function in Matlab 6.5. The generalized Hurst index of all the four groups of data is computed. The parameters are set like this: $s=20,21, \ldots, 50 ; q=-10,-9.5,-9, \ldots, 9,9.5$, $10(q \neq 0) ; k=1,2,3$. The range of $s$ is from 20 to 50 since too many data points may cause over fitting and insufficient data point cannot give enough information. Table 1 gives the simulation result when $k=2$ (MF-DFA2).

It is shown in Table 1 when $q$ increases from -10 to 10 , the generalized Hurst index $H(q)$ decreases from 0.463 to 0.215 for data from Handan Steel, 1.115 to 0.530 for data from Laiwu Steel and 1.021 to 0.379 for data from Linfen Steel. For the generated random series, the range of $H(q)$ is between 0.539 and 0.497 . The results show that $H(q)$ and $q$ 
is significantly uncorrelated for the random sequence, while for the three blast furnaces with different volumes, they are significantly correlated. $H(q)$ and $q$ being significantly uncorrelated suggests that $F_{q}(s)$ is uncorrelated with $q$, e.g., the $q$-order fluctuation is the same for every section of a detrended time series. Thus the time series has uniform local structure and is ideally single fractal. While $H(q)$ and $q$
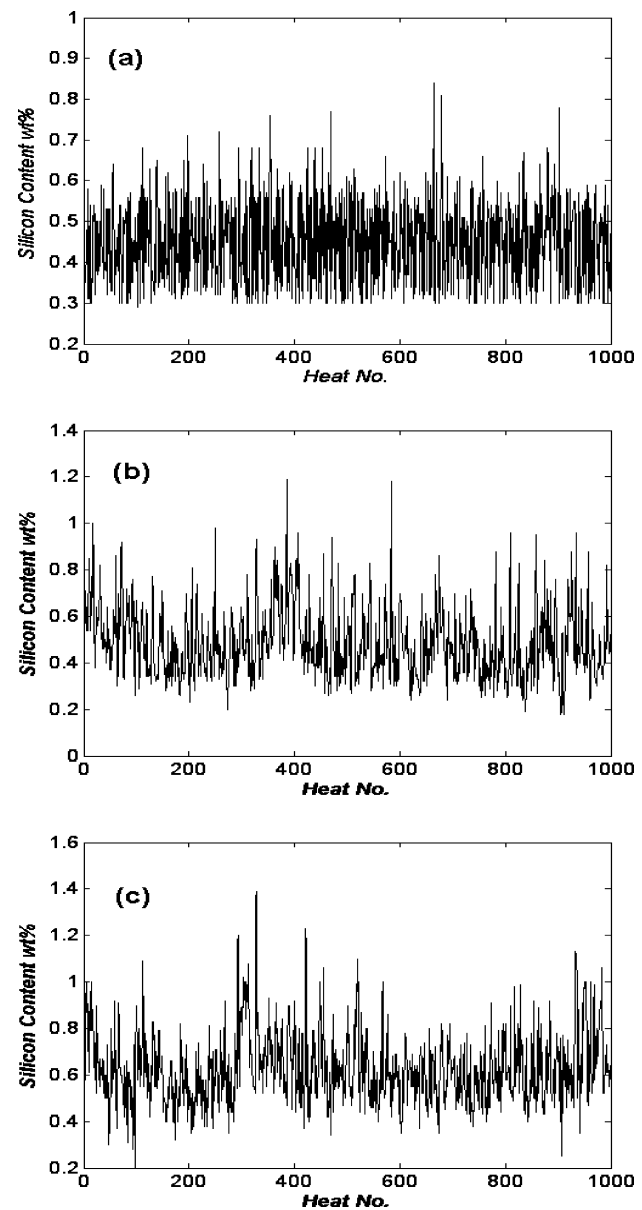

Fig. 1. Time series of silicon content in hot metal at No. $7 \mathrm{BF}$ at Handan Steel (a), No. 1 BF at Laiwu Steel (b) and No. 6 $\mathrm{BF}$ at Linfen Steel (c). being significantly correlated suggests $F_{q}(s)$ is correlated with $q$, the local structure of time series is not uniform and such series is multi-fractal.

Figure 2 gives the generalized Hurst index $H(q)$ for the three blast furnaces. It is shown that the generalized Hurst index $H(q)$ declines with the increase of $q$. Based on the discovery, we can conclude $F_{q}(s)$ is related to $q$. That is to say, the local structure of silicon content series is not uniform since the detrended fluctuation of power $q$ in section $v_{s}$ is different. Thus the multi-fractal characteristics of silicon content series for all the three Blast Furnaces are confirmed. For the random generated series, $H(q)$ fluctuates around 0.5 , the local structure of such a series is uniform and this structure is single fractal. Theoretically $H(q)$ for a random series is 0.5 . Since the random series generated by Matlab 6.5 is pseudo-random, it is normal for fluctuation between 0.497 and 0.539 .

It is also shown that the range of fluctuation for Han Steel is around 0.25, the minimum of the three Blast Furnaces. The range for Laiwu Steel is around 0.59 and for Linfen Steel about 0.64. Comparatively, there are more local "fluctuation levels" for a blast furnace with relatively small volume and the multi-fractal characteristics are more obvious. The control of silicon content is difficult since the probability of abrupt transition is larger.

As a whole, the silicon content series reflect single fractal characteristics, but locally there are remarkable multifractal characteristics. It is very difficult to fit and predict such a process using regular methods. Research efforts based on nonlinear analysis like fractal and chaos should be a promising direction.

Figures 3, 5 and 7 give the scale function $\tau(q)$ for the three silicon content series. It is seen that the scale function is nonlinear and upper convex. That is another evidence of the existence of multi-fractal characteristic. Figures 4, 6 and $\mathbf{8}$ also give the estimation results of multi-fractal spectrum $f(a)$. The curve described by "*" is a rough graph of $f(a)$. The local Holder index also has a high fluctuation level, which is another proof of the multi-fractal characteristics.

Table 1. Numerical results of generalized Hurst index for silicon content series and the random series (only when $q$ is integer listed).

\begin{tabular}{||c|c|c|c|c|c|c|c|c|c|c||}
\hline $\mathbf{q}$ & $\mathbf{- 1 0}$ & $\mathbf{- 9}$ & $\mathbf{- 8}$ & $\mathbf{- 7}$ & $\mathbf{- 6}$ & $\mathbf{- 5}$ & $\mathbf{- 4}$ & $\mathbf{- 3}$ & $\mathbf{- 2}$ & $\mathbf{- 1}$ \\
\hline $\begin{array}{c}\text { Han } \\
\text { No.7 }\end{array}$ & 0.463 & 0.459 & 0.455 & 0.450 & 0.444 & 0.437 & 0.429 & 0.421 & 0.411 & 0.40 \\
\hline $\begin{array}{c}\text { Lai } \\
\text { No.1 }\end{array}$ & 1.115 & 1.098 & 1.077 & 1.049 & 1.014 & 0.970 & 0.920 & 0.866 & 0.812 & 0.762 \\
\hline $\begin{array}{c}\text { Lin } \\
\text { No.6 }\end{array}$ & 1.021 & 1.007 & 0.989 & 0.966 & 0.939 & 0.907 & 0.870 & 0.832 & 0.796 & 0.764 \\
\hline Randn & 0.525 & 0.517 & 0.510 & 0.503 & 0.499 & 0.497 & 0.498 & 0.502 & 0.508 & 0.517 \\
\hline \hline q & $\mathbf{1}$ & $\mathbf{2}$ & $\mathbf{3}$ & $\mathbf{4}$ & $\mathbf{5}$ & $\mathbf{6}$ & $\mathbf{7}$ & $\mathbf{8}$ & $\mathbf{9}$ & $\mathbf{1 0}$ \\
\hline $\begin{array}{c}\text { Han } \\
\text { No.7 }\end{array}$ & 0.375 & 0.359 & 0.343 & 0.324 & 0.305 & 0.285 & 0.266 & 0.247 & 0.231 & 0.215 \\
\hline $\begin{array}{c}\text { Lai } \\
\text { No.1 }\end{array}$ & 0.676 & 0.640 & 0.610 & 0.585 & 0.567 & 0.554 & 0.544 & 0.538 & 0.533 & 0.530 \\
\hline $\begin{array}{c}\text { Lin } \\
\text { No.6 }\end{array}$ & 0.703 & 0.662 & 0.612 & 0.559 & 0.511 & 0.471 & 0.439 & 0.414 & 0.394 & 0.379 \\
\hline Randn & 0.534 & 0.538 & 0.539 & 0.536 & 0.531 & 0.524 & 0.517 & 0.510 & 0.504 & 0.498 \\
\hline \hline
\end{tabular}



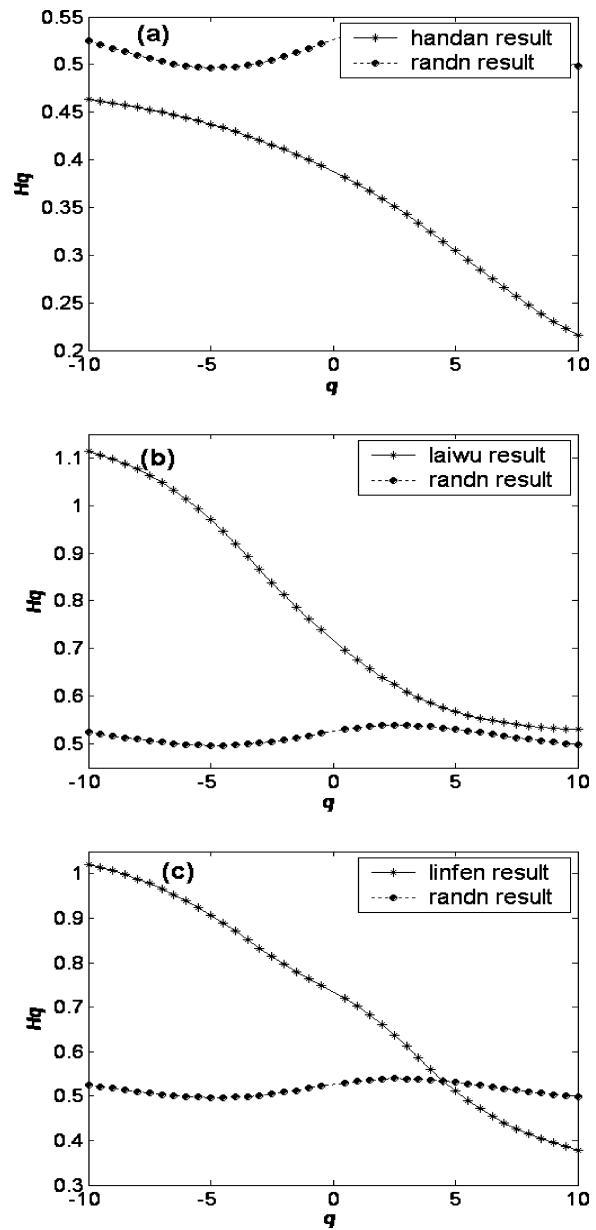

Fig. 2. (a), (b), (c) are the comparative graphs between the generalized Hurst index $H(q)$ for $7 \#$ BF at Handan Steel, 1\# BF at Laiwu Steel, 6\# BF at Linfen Steel and $H(q)$ for the random series respectively.

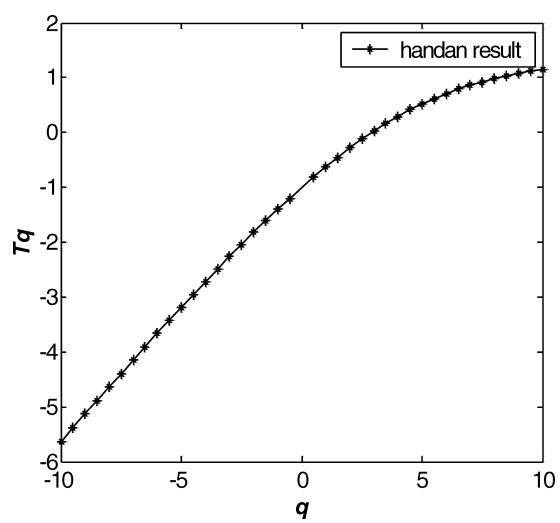

Fig. 3. The numerical result for the estimation of scale function for data from 7\# BF at Handan steel.

\section{Conclusions}

This paper gives a tentative research on the multi-fractal characteristics of time series of silicon content in hot metal to describe the local features of the fluctuation of silicon content. We have attempted to introduce some concepts and methodologies associated to the analysis of fractal time series. Several minor techniques are applied to make the model more robust. Computation of generalized Hurst index, scale function and multi-fractal spectrum shows the

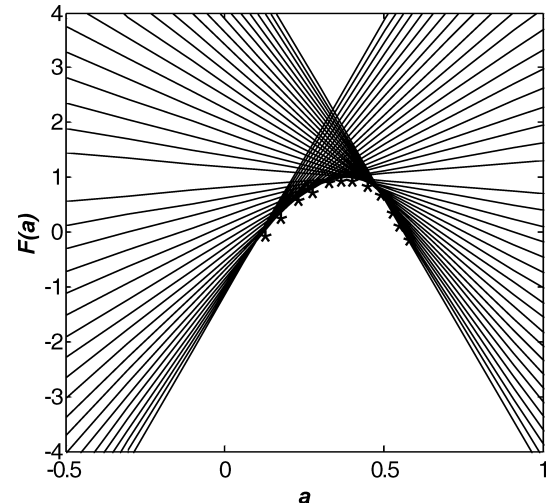

Fig. 4. The numerical result for the estimation of the multi-fractal spectrum for data from 7 \# BF at Handan steel.

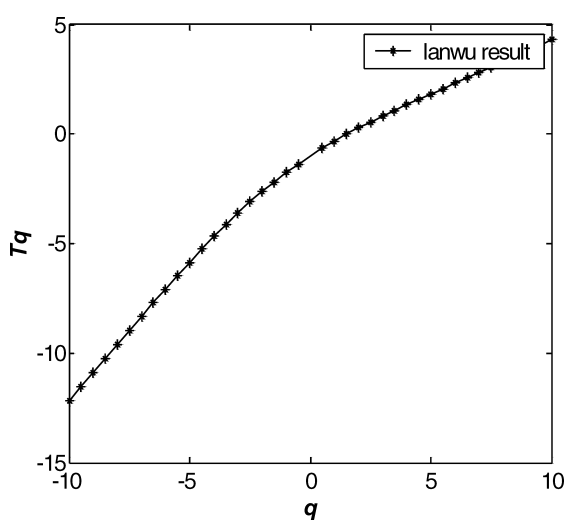

Fig. 5. The numerical result for the estimation of scale function for data from 1 \# BF at Laiwu steel.

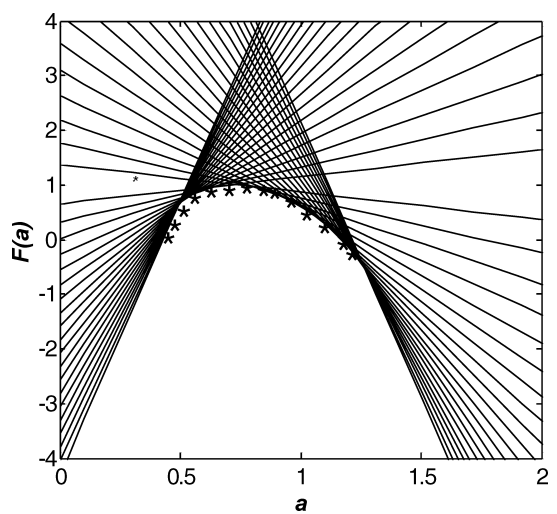

Fig. 6. The numerical result for the estimation of the multi-fractal spectrum for data from 1 \# BF at Laiwu steel.

time series of silicon content in hot metal at three blast furnaces are multi-fractal. It is also concluded time series of silicon content from blast furnaces with smaller volume show stronger multi-fractal characteristics and the probability of abrupt transition is larger.

For a multi-fractal time series, the previous research based on normal distribution, series uncorrelated and single fractal needs amendment and improvement. Future work should be done on the following fields:

First, By combining previous predictive models with local parameters got through multi-fractal identification, models with more accuracy, stability and robustness can be expected. Second, extend the multi-fractal methodologies to 


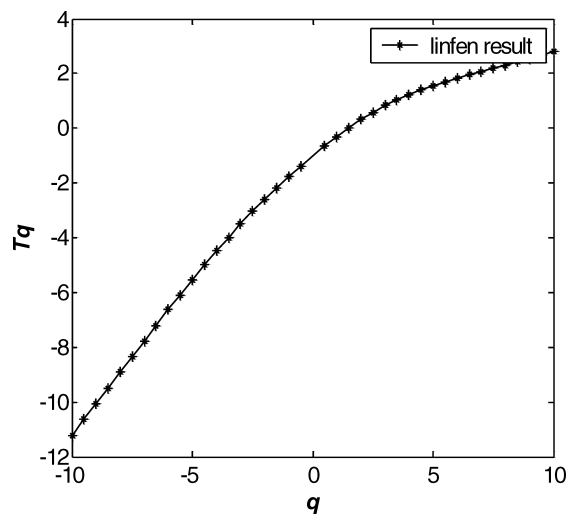

Fig. 7. The numerical result for the estimation of scale function for data from 6\# BF at Linfen Steel.

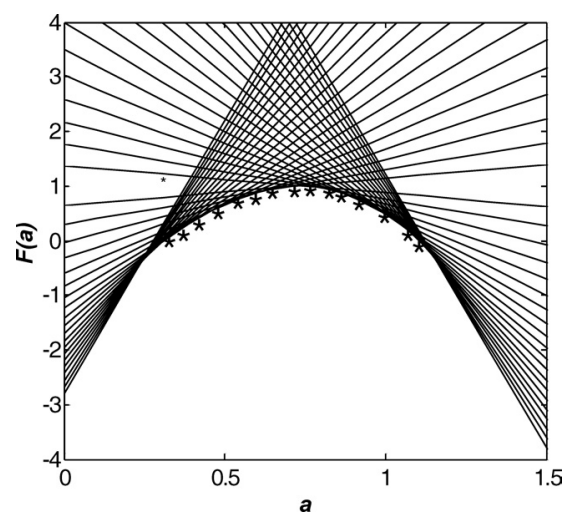

Fig. 8. The numerical result for the estimation of the multi-fractal spectrum for data from 6 \# BF at Linfen Steel.

the analysis of other important variables in blast furnace iron-making such as wind blasted, coke rate and so on, a closed-loop control model can be set up with the critical control variables and state variables.

\section{Acknowledgements}

The authors wish to thank the Ministry of Science and
Technology Foundation of China (No. 2005EC000166) and Ningbo Natural Science Foundation (No. 2006A610032) due to the financial support.

\section{REFERENCES}

1) I. Muchi, J. Yagi, K. Tamura and A. Moriyama: J. Jpn. Inst. Met., 4 (1966), 826.

2) S. Amano, T. Takarabe, T. Nakamori and etc.: ISIJ Int., 30 (1990), 105.

3) L. K. Chen, H. B. Zuo, Z. J. Yu and T. J. Yang: Iron Steel (China), 41 (2006), 14.

4) X. G. Liu and F. Liu: Optimization and Intelligent Control System of Blast Furnace Ironmaking Process, Metallurgical industry Press, Beijing, (2003), 15.

5) S. P. Mehrotra and Y. C. Nand: ISIJ Int., 33 (1993), 839.

6) H. Saxen: Can. Metall. O., 33 (1994), 319.

7) H. Singh, N. V. Sridhar and B. Deo: Steel Res., 67 (1996), 521.

8) P. R. Austin, H. Nogami and J. Yagi: ISIJ Int., 38 (1998), 246.

9) T. Miyano, S. Kimoto, H. Shibuta, K. Nakashima, Y. Ikenaga and K. Aihara: Phy. D, 135 (2000), 305

10) J. Chen: Eng. Appl. Artif. Intell., 14 (2001), 77.

11) J. A. Castro, H. Nogami and J. Yagi: ISIJ Int., 42 (2002), 44

12) M. Waller and H. Saxen: ISIJ Int., 42 (2002), 316.

13) J. Jiménez, J. Mochón, J. S. Ayala and F. Obeso: ISIJ Int., 44 (2004), 573.

14) C. H. Gao and J. X. Qian: ISIJ Int., 45 (2005), 1269.

15) S. H. Luo and X. G. Liu: Acta Phys. Sin. (China), 55 (2006), 3343.

16) P. Grassberger: Phys. Lett. A, 97 (1983), 227.

17) T. C. Halsey and M. H. Jensen: Phys. Rev. A, 33 (1986), 1141.

18) E Bacry, J. Delour and J. F. Muzy: Phys. Rev. E, 64 (2001), 026103.

19) J. W. Kantelhardt, A. Z. Stephan and K. B. Eva: Phys. A, 316 (2002), 87.

20) R. Benzi and G. Paladin: J. Phys., 17A (1984), 3521.

21) A. Fisher, L. Calvet and B. Mandelbrot: Yale University, Working Paper, (1997), 1.

22) B. Mandelbrot, A. Fisher and L. Calvet: Yale University, Working Paper, (1997), 1.

23) L. Calvet, A. Fisher and B. Mandelbrot: Yale University, Working Paper, (1997), 1.

24) T. C. Halsey and M. H. Jensen: Phys. Rev. A, 33 (1986), 1141.

25) Z. Y. Wen, A. H. Fan and Z. X. Wen: The Theory and Applications of Fractal, Zhejiang Technology and Science Press, Hangzhou, (1998), 7. 